\title{
Minimal Algorithms for Lipschitz Monoids and Vahlen Monoids
}

\author{
Jacques Helmstetter ${ }^{1}$ \\ ${ }^{1}$ Institut Fourier, Université de Grenoble I, France \\ Correspondence: Jacques Helmstetter, Institut Fourier, B.P. 74, 38402 Saint-Martin d'Hères, France. E-mail: \\ jacques.helmstetter@ujf-grenoble.fr
}

Received: May 29, 2013 Accepted: June 19, 2013 Online Published: October 25, 2013

doi:10.5539/jmr.v5n4p39 URL: http://dx.doi.org/10.5539/jmr.v5n4p39

\begin{abstract}
Every Clifford algebra $\mathrm{C} \ell(V, q)$ contains a $\operatorname{Lipschitz}$ monoid $\operatorname{Lip}(V, q)$, which is in general (but not always) the multiplicative monoid generated by all vectors; its even and odd components are closed irreducible algebraic submanifolds. In this article, an algorithm allows to decide whether a given even or odd element of $\mathrm{C} \ell(V, q)$ belongs to $\operatorname{Lip}(V, q)$; it is minimal because the number of required verifications is equal to the codimension of the even and odd components of $\operatorname{Lip}(V, q)$. There is an immediate application to Vahlen matrices, since the Vahlen monoid is the image of a Lipschitz monoid.
\end{abstract}

Keywords: Clifford algebras, Lipschitz monoids, Vahlen matrices

Let $V$ be a vector space of finite dimension $n \geq 1$ over a field $K$, let $q$ be a quadratic form $V \rightarrow K$, and $\mathrm{b}_{q}$ the associated bilinear form $V \times V \rightarrow K$ :

$$
\mathrm{b}_{q}(x, y)=q(x+y)-q(x)-q(y)
$$

although the field $K$ will later be especially the field $\mathbb{R}$ of real numbers, the preliminary theory is valid for every field $K$, even for a field of characteristic 2 that does not allow to define the polar form $\mathrm{b}_{q} / 2$. Let $\mathrm{C} \ell(V, q)$ be the associated Clifford algebra, that is the associative and unital $K$-algebra generated by the elements $x \in V$ with the relations $x^{2}=q(x)$ (whence $x y+y x=\mathrm{b}_{q}(x, y)$ ). The reversion $\rho$ is the involutive anti-automorphism of $\mathrm{C} \ell(V, q)$ that extends the identity mapping of $V$, and the grade automorphism $\sigma$ is the involutive automorphism that extends $x \longmapsto-x$. The name of $\sigma$ refers to the parity gradation $\mathrm{C} \ell(V, q)=\mathrm{C} \ell_{0}(V, q) \oplus \mathrm{C} \ell_{1}(V, q)$, where $\mathrm{C} \ell_{0}(V, q)$ is the even subalgebra, generated by all $x y$, and $\mathrm{C} \ell_{1}(V, q)$ the odd subspace which contains $V$; the dimension of each component is $2^{n-1}$.

When $\left(e_{1}, \ldots, e_{n}\right)$ is a basis of $V$, there is a basis of $\mathrm{C} \ell(V, q)$ made of all products $e_{j_{1}} e_{j_{2}} \cdots e_{j_{k}}$ such that $0 \leq k \leq n$ and $j_{r}<j_{r+1}$ for $r=1,2, \ldots, k-1$ (if $k \geq 2$ ). For such a product, we will often use the short notation $e_{j_{1}, j_{2}, \ldots, j_{k}}$ (only allowed if $j_{1}<j_{2}<\ldots<j_{k}$ ), or $e_{J}$ (where $J=\left\{j_{1}, j_{2}, \ldots, j_{k}\right\}$ ); this product means 1 when $k=0$ and $J=\emptyset$.

The Lipschitz monoid (or Lipschitz semi-group) $\operatorname{Lip}(V, q)$ shall be defined in Section 2; in general, it is the multiplicative monoid generated in $\mathrm{C} \ell(V, q)$ by the vectors $x \in V$; its elements are called lipschitzian elements. Its even (resp. odd) component $\operatorname{Lip}_{0}(V, q)\left(\operatorname{resp} . \operatorname{Lip}_{1}(V, q)\right)$ is a closed irreducible algebraic submanifold of $\mathrm{C} \ell_{0}(V, q)$ (resp. $\left.\mathrm{C} \ell_{1}(V, q)\right)$, of dimension $1+\frac{1}{2} n(n-1)$ (when its dimension can be defined). When $a$ is lipschitzian, then $\rho(a)$ is lipschitzian too, and commutes with $a$, the product $a \rho(a)$ is a scalar, and $a z \rho(a)$ is a vector for every $z \in V$; $a$ is invertible if and only if $a \rho(a) \neq 0$; and when it is invertible, it determines an orthogonal transformation $x \longmapsto \pm a x a^{-1}$ of $(V, q)$; the sign \pm means + or - according as $a$ is even or odd.

The first problem tackled in this paper is this one: if $a$ is a given even or odd element of $\mathrm{C} \ell(V, q)$, how can we find out whether $a$ is lipschitzian? When $n \leq 3$, the answer is always positive because $\operatorname{Lip}_{p}(V, q)=\mathrm{C} \ell_{p}(V, q)$ for each parity $p$. When $n=4$, let us use a basis $\left(e_{1}, e_{2}, e_{3}, e_{4}\right)$ of $V$; there are eight scalars $\kappa, \kappa^{\prime}, \lambda, \lambda^{\prime}, \mu, \mu^{\prime}, v, v^{\prime} \in K$ such that, according to the parity of $a$,

$$
a=\kappa+\lambda e_{1,2}+\mu e_{1,3}+v e_{1,4}+v^{\prime} e_{2,3}+\mu^{\prime} e_{2,4}+\lambda^{\prime} e_{3,4}+\kappa^{\prime} e_{1,2,3,4},
$$

or

$$
a=\kappa e_{1}+\lambda e_{2}+\mu e_{3}+v e_{4}+v^{\prime} e_{1,2,3}+\mu^{\prime} e_{1,2,4}+\lambda^{\prime} e_{1,3,4}+\kappa^{\prime} e_{2,3,4} ;
$$


as explained in Section 4, $a$ is lipschitzian if and only if

$$
\kappa \kappa^{\prime}-\lambda \lambda^{\prime}+\mu \mu^{\prime}-v v^{\prime}=0 .
$$

The calculation of $a \rho(a)$ (which is very toilsome if the basis $\left(e_{1}, e_{2}, e_{3}, e_{4}\right)$ is not orthogonal) would show that $(0.1)$ is equivalent to $a \rho(a) \in K$. This Equation (0.1) is valid for all bases of $V$ and does not involve $q$. In Section 4, these features are confirmed for every dimension $n \geq 5$ : if we use a basis $\left(e_{1}, \ldots, e_{n}\right)$ of $V$, and the derived basis $\left(e_{J}\right)$ of $\mathrm{C} \ell(E, q)$ (where $J$ runs through the set $\mathcal{P}(N)$ of all subsets of $N=\{1,2, \ldots, n\}$ ), then the quadratic form $q$ is not involved in the answer, which even ignores whether $q$ is degenerate or not. The answer follows from an algorithm that is minimal in the sense of Section 1: the number of necessary verifications is the codimension $2^{n-1}-1-\frac{1}{2} n(n-1)$ of $\operatorname{Lip}_{p}(V, q)$ in $\mathrm{C} \ell_{p}(V, q)$ (for $\left.p=0,1\right)$.

This minimal algorithm is presented in Section 4; it is based on the invariance theorem which is presented in Section 3. Examples follow in Section 5.

This study has an immediate application to Vahlen matrices; this is explained in Sections 6 and 7, and examples follow in Section 8.

\section{Minimal Algorithms}

Let $E$ be a vector space of dimension $d$ over $K$, with a basis $\left(\epsilon_{1}, \ldots, \epsilon_{d}\right)$, and let $M$ be a closed algebraic submanifold of codimension $c$ in $E$. A minimal algorithm for $M$ is an algorithm that allows to find out whether an element $a=\sum_{k=1}^{d} a_{k} \epsilon_{k}$ of $E$ belongs to $M$ just by verifying the vanishing at the point $\left(a_{1}, \ldots, a_{d}\right)$ of $c$ polynomial functions $K^{d} \rightarrow K$.

For instance, let $V$ be a vector space of dimension $n \geq 3$ provided with a basis $\left(e_{1}, \ldots, e_{n}\right)$, and let $M$ be the subset of all $(a, b) \in V \times V$ such that $a$ and $b$ are colinear vectors in $V$; here, $M$ is a closed algebraic manifold of codimension $n-1$ in $V \times V$. The following well known theorem gives a minimal algorithm for $M$ in $V \times V$.

Theorem 1.1 Let $a=\sum_{k=1}^{n} a_{k} e_{k}$ and $b=\sum_{k} b_{k} e_{k}$ be two vectors in $V$ such that $\left(a_{j}, b_{j}\right) \neq(0,0)$ for some $j \in\{1,2, \ldots, n\}$. These two vectors are colinear if and only if $a_{j} b_{k}-b_{j} a_{k}=0$ for all $k$ other than $j$.

The preliminary condition $\left(a_{j}, b_{j}\right) \neq(0,0)$ is important; although all equations $a_{1} b_{k}-b_{1} a_{k}=0$ are satisfied when $a=e_{2}$ and $b=e_{3}$, the violation of the condition $\left(a_{1}, b_{1}\right) \neq 0$ prevents us from concluding that $e_{2}$ and $e_{3}$ are colinear. Most minimal algorithms require such preliminary conditions, and there must be enough of such conditions to foresee all possible cases.

It is easy to show that the classical algorithm for lipschitzian elements is not minimal; moreover, it requires the given element $a \in \mathrm{C} \ell(V, q)$ to be invertible, and $q$ to be nondegenerate (or at least, $\operatorname{dim}\left(\operatorname{ker}\left(\mathrm{b}_{q}\right)\right) \leq 3$ ). It is based on this statement: $a$ is an invertible lipschitzian element if an only if $a$ is even or odd, $a \rho(a)$ is an invertible scalar, and $a z \rho(a)$ is a vector for every $z \in V$. An orthogonal basis $\left(e_{1}, \ldots, e_{n}\right)$ of $V$ much simplifies the application of this classical algorithm, because on one side, $\rho\left(e_{J}\right)= \pm e_{J}$ (see (2.7) below), and on the other side, $\rho$ leaves invariant $a \rho(a)$ and $a z \rho(a)$. Thus, to verify $a \rho(a) \in K$, the number of verifications is $\left(\begin{array}{c}n \\ 4\end{array}\right)+\left(\begin{array}{c}n \\ 8\end{array}\right)+\cdots$; and to verify $a e_{j} \rho(a) \in V$ (for one index $j$ ), the number of verifications is $\left(\begin{array}{c}n \\ 5\end{array}\right)+\left(\begin{array}{c}n \\ 9\end{array}\right)+\cdots$. When $n=4,5,6,7,8, \ldots$, the number of necessary verifications is equal to $1,10,51,182,519, \ldots$, much more than the codimension $1,5,16,42,99, \ldots$ of $\operatorname{Lip}_{p}(V, q)$ in $\mathrm{C} \ell_{p}(V, q)$. Moreover, the complete calculation of $a \rho(a)$ is necessary to verify that $a$ is invertible; and the invertibility of $a$ is really indispensable, as it appears in the following counter-example, where $\left(e_{1}, \ldots, e_{6}\right)$ is an orthogonal basis such that $q\left(e_{3}\right)=q\left(e_{4}\right)=1$ and $q\left(e_{5}\right)=q\left(e_{6}\right)=-1$ :

$$
\begin{aligned}
a & =\left(1+e_{1,2,3,4}\right)\left(e_{3}+e_{5}\right)\left(e_{4}+e_{6}\right) \\
& =-e_{1,2}+e_{3,4}+e_{3,6}-e_{4,5}+e_{5,6}-e_{1,2,3,5}-e_{1,2,4,6}+e_{1,2,3,4,5,6}
\end{aligned}
$$

in Section 5, it shall be proved that this $a$ is not lipschitzian; nevertheless, $a \rho(a)=0$, and $a z \rho(a)=0$ or all $z \in V$.

\section{Lipschitz Monoids}

The following definition is justified by all the simple and effective theorems that it allows to state.

Definition 2.1 The Lipschitz monoid (or Lipschitz semi-group) $\operatorname{Lip}(V, q)$ is the multiplicative monoid generated in $\mathrm{C} \ell(V, q)$ by all scalars in $K$, all vectors in $V$ and all $1+x y$ where $x$ and $y$ are vectors such that $q(x)=q(y)=$ $\mathrm{b}_{q}(x, y)=0$ (but $x y \neq 0$ ). It is the union of its intersections $\operatorname{Lip}_{0}(V, q)$ and $\operatorname{Lip}_{1}(V, q)$ with $\mathrm{C} \ell_{0}(V, q)$ and $\mathrm{C} \ell_{1}(V, q)$.

Definition 2.1 is meaningful even when $q$ vanishes everywhere. When $q=0$, then $C \ell(V, 0) \cong \wedge(V)$, and it is clear that $\operatorname{Lip}(V, 0)$ needs the three kinds of generators mentioned in Definition 2.1. When there is a vector $z$ such that 
$q(z) \neq 0$, then every scalar $\lambda$ is the product of $\lambda z$ and $z^{-1}=z / q(z)$. When the plane spanned by two vectors $x$ and $y$ contains an invertible vector $z$, it is easy to prove that $(1+x y) z$ is a vector, and that $1+x y$ is a product of two vectors. When $q(x)=q(y)=\mathrm{b}_{q}(x, y)=0$, it is more difficult to prove that $1+x y$ is a product of four vectors, provided that $V$ contains an invertible vector, and that $K$ contains at least three elements. Although we are here interested in the field $\mathbb{R}$, and not in $\mathbb{Z} / 2 \mathbb{Z}=\{0,1\}$, the importance of Definition 2.1. justifies the statement a precise theorem (Helmstetter \& Micali, 2008).

Theorem 2.2 The vectors of $V$ are sufficient to generate the monoid $\operatorname{Lip}(V, q)$ except in these three cases:

(1) when $q=0$;

(2) when $K \cong \mathbb{Z} / 2 \mathbb{Z}, \operatorname{dim}(V) \geq 4$, and $V$ contains a basis $\left(e_{1}, e_{2}, \ldots\right)$ such that $q\left(\sum_{j} \lambda_{j} e_{j}\right)=\lambda_{1} \lambda_{2}+\lambda_{3} \lambda_{4}$. In this case (Dieudonné's exceptional case), $\operatorname{Lip}(V, q)$ is generated by the vectors and $1+e_{2} e_{3}$.

(3) when $K \cong \mathbb{Z} / 2 \mathbb{Z}, \operatorname{dim}(V) \geq 3$, and $V$ contains a basis $\left(e_{1}, e_{2}, \ldots\right)$ such that $q\left(\sum_{j} \lambda_{j} e_{j}\right)=\lambda_{1} \lambda_{2}$. In this case, $\operatorname{Lip}(V, q)$ is generated by the vectors and all $1+e_{2} e_{j}$ with $j \geq 3$.

Definition 2.1 ensures the equalities $\operatorname{Lip}_{p}(V, q)=\mathrm{C} \ell_{p}(V, q)$ when $n \leq 3$; and when $n=4$, it ensures that the Equation (0.1) is necessary and sufficient for $a$ to be lipschitzian, even if $q=0$. Moreover, it ensures the following theorem (Helmstetter, 2005), which involves a subspace $U$ of $V$; the notation $\mathrm{C} \ell(U, q)$ means the subalgebra of $\mathrm{C} \ell(V, q)$ generated by $U$ (and the unit element, indispensable if $U$ is totally isotropic); it is canonically isomorphic to the Clifford algebra of the restriction of $q$ to $U$.

Theorem 2.3 An element of $\mathrm{C} \ell(U, q)$ is lipschitzian in $\mathrm{C} \ell(V, q)$ if and only if it is lipschitzian in $\mathrm{C} \ell(U, q)$.

From Definition 2.1, it immediately follows that $\operatorname{Lip}(V, q)$ is invariant by $\rho$, that $a \rho(a)=\rho(a) a \in K$ and $a z \rho(a) \in V$ for all $a \in \operatorname{Lip}(V, q)$ and all $z \in V$. Consequently, the $\operatorname{subset} \operatorname{GLip}(V, q)$ of all invertible lipschitzian elements is a group, and each element $a$ of this group gives an orthogonal transformation $z \longmapsto \pm a z a^{-1}$ of $(V, q)$. In this way we obtain all orthogonal transformations $g$ of $V$ such that firstly, $q(g(z))=q(z)$ for all $z \in V$, and secondly (when $q$ is degenerate), $g(z)=z$ for all $z \in \operatorname{ker}\left(\mathrm{b}_{q}\right)$. When $\operatorname{dim}(V)$ is infinite, these conditions on $g$ must be strengthened (Helmstetter, 2005; Helmstetter \& Micali, 2008; Helmstetter, 2012).

The neutral $\operatorname{Lipschitz~monoid} \operatorname{Lip}(V, 0)$ shall play a capital role, and we need a precise description of $\operatorname{Lip}(V, 0)$. Since $C \ell(V, 0) \cong \bigwedge(V)$, the neutral algebra $C \ell(V, 0)$ is graded over the group $\mathbb{Z}$, and we can define the space $\mathrm{C} \ell^{2}(V, 0)$ of bivectors, which is spanned by all products $x y$ of two vectors; since every $u \in \mathrm{C} \ell^{2}(V, 0)$ is nilpotent, we can define $\exp (u)$ in the commutative algebra $\mathrm{C} \ell_{0}(V, q)$; if $u=x_{1} y_{1}+x_{2} y_{2}+\cdots+x_{r} y_{r}$ (for some $r \geq 1$ ), then

$$
\exp \left(x_{1} y_{1}+x_{2} y_{2}+\cdots+x_{r} y_{r}\right)=\left(1+x_{1} y_{1}\right)\left(1+x_{2} y_{2}\right) \cdots\left(1+x_{r} y_{r}\right)
$$

this equality eschews the exponential series and determines a mapping exp: $\mathrm{C} \ell^{2}(V, 0) \rightarrow \mathrm{C} \ell_{0}(V, 0)$ even when $K$ is a field of characteristic $\neq 0$ (Chevalley, 1954). To describe $\operatorname{Lip}(V, 0)$, we also need the decomposable elements, which are the elements of the multiplicative monoid generated in $\mathrm{C} \ell(V, 0)$ by the scalars and the vectors.

Theorem 2.4 An element $a \in \mathrm{C} \ell(V, 0)$ is lipschitzian if and only if $a=d \exp (u)$ for some decomposable element $d$ and for some $u \in \mathrm{C} \ell^{2}(V, 0)$.

The forthcoming calculations are easier with the following notation. The cardinal of a subset $F$ of $N$ is denoted by $|F|$. The boolean sum $F+G$ of two subsets is $F \cup G \backslash F \cap G$; thus $|F+G| \equiv|F|+|G|$ modulo 2 . With the boolean addition, $\mathcal{P}(N)$ becomes a vector space of dimension $n$ over $\mathbb{Z} / 2 \mathbb{Z}$, with basis $(\{1\},\{2\}, \ldots,\{n\})$. Let $S$ : $\mathcal{P}(N) \times \mathcal{P}(N) \rightarrow \mathbb{Z} / 2 \mathbb{Z}$ be the bilinear form such that $S(\{i\},\{j\})$ is equal to 0 modulo 2 if $i \leq j$, to 1 modulo 2 if $i>j$. When $|F|=f$ and $|G|=g$, this definition implies

$$
\begin{gathered}
S(G, F)-S(F, G)=f g+|F \cap G| \text { modulo } 2, \\
S(F, F)=\frac{1}{2} f(f-1) \text { modulo } 2 .
\end{gathered}
$$

Let us calculate $\exp (u)$ when $u$ is given by its coordinates $u_{J}$ (with $|J|=2$ ) in the basis $\left(e_{J}\right)$ derived from some basis $\left(e_{1}, \ldots, e_{n}\right)$ of $V$. Let us write $a=\exp (u)=\sum_{J} a_{J} e_{J}$ with a sum running on all subsets $J$ of even cardinal. Obviously $a_{\emptyset}=1$ and $a_{J}=u_{J}$ when $|J|=2$. Let $J$ be a subset of even cardinal $\geq 2$, and let $i$ be an element of $J$; it is convenient, but not obligatory, to choose the smallest element of $J$; from (2.1), it follows that

$$
a_{J}=\sum_{j \in J+\{i\}}(-1)^{r} u_{\{i, j\}} a_{J+\{i, j\}},
$$


where $r$ is the number of elements of $J$ between $i$ and $j$ (the number of $k \in J$ such that $i<k<j$ if $i<j$, such that $j<k<i$ if $i>j$ ). Since $J+\{i\}=J \backslash\{i\}$, the sum runs on all $j \in J$ other than $i$; and $u_{\{i, j\}}$ means either $u_{i, j}$ if $i<j$, or $u_{j, i}$ if $i>j$. This equality allows to deduce all $a_{J}$ from $a_{\emptyset}=1$, when all $u_{J}$ (with $|J|=2$ ) are known.

When $i$ and $j$ belong to $J$, it is easy to verify that the parity of the number $r$ of elements of $J$ between $i$ and $j$ is equal to $S(\{i, j\}, J+\{i, j\})=S(\{i, j\}, J)+1$. This fact allows to improve (2.4):

$$
a_{J}=-\sum_{j \in J+\{i\}}(-1)^{S(\{i, j\}, J)} u_{\{i, j\}} a_{J+\{i, j\}} .
$$

The above bilinear form $S$ is also useful in another context: if the basis $\left(e_{1}, \ldots, e_{n}\right)$ is orthogonal, then for all $F, G \in \mathcal{P}(N)$,

$$
\begin{gathered}
e_{F} e_{G}=(-1)^{S(F, G)} e_{F+G} \prod_{j \in F \cap G} q\left(e_{j}\right), \\
\rho\left(e_{F}\right)=(-1)^{S(F, F)} e_{F} .
\end{gathered}
$$

\section{The Invariance Theorem}

The invariance theorem states that the Lipschitz monoids are invariant by deformation, and the concept of deformation needs the preliminary definition of interior multiplications. Beside $(V, q)$, we still consider two spaces $U$ and $W$ and two bilinear mappings $\eta: U \times V \rightarrow K$ and $\theta: V \times W \rightarrow K$. There are two bilinear mappings (called interior multiplications)

$$
\begin{array}{ll}
\bigwedge(U) \times \mathrm{C} \ell(V, q) \rightarrow \mathrm{C} \ell(V, q), & (a, b) \longmapsto a\rfloor_{\eta} b, \\
\mathrm{C} \ell(V, q) \times \bigwedge(W) \rightarrow \mathrm{C} \ell(V, q), & (b, c) \longmapsto b_{\theta}\lfloor c,
\end{array}
$$

satisfying the following properties for all $a, a^{\prime} \in \bigwedge(U)$, all $b, b^{\prime} \in \mathrm{C} \ell(V, q)$, all $c, c^{\prime} \in \bigwedge(W)$, and for all $x \in U$, $y \in V$ and $z \in W$ :

$$
\begin{array}{lr}
1\rfloor_{\eta} b=b, & b_{\theta}\lfloor 1=b, \\
\left.\left.\left.\left(a \wedge a^{\prime}\right)\right\rfloor_{\eta} b=a\right\rfloor_{\eta}\left(a^{\prime}\right\rfloor_{\eta} b\right), & b_{\theta}\left\llcorner\left(c \wedge c^{\prime}\right)=\left(b _ { \theta } \lfloor c ) _ { \theta } \left\lfloorc^{\prime},\right.\right.\right. \\
x\rfloor_{\eta} y=\eta(x, y), & y_{\theta}\lfloor z=\theta(y, z), \\
\left.\left.x\rfloor_{\eta} b b^{\prime}=(x\rfloor_{\eta} b\right) b^{\prime}+\sigma(b)(x\rfloor_{\eta} b^{\prime}\right), & b b^{\prime}{ }_{\theta}\left\lfloor z=b\left(b^{\prime}{ }_{\theta}\lfloor z)+\left(b b_{\theta}\lfloor z) \sigma\left(b^{\prime}\right) .\right.\right.\right.
\end{array}
$$

The first two lines, together with $\left.\left.(a\rfloor_{\eta} b\right)_{\theta} \mathrm{L} c=a\right\rfloor_{\eta}\left(b_{\theta} \mathrm{L} c\right)$, mean that the space $\mathrm{C} \ell(V, q)$ is a bimodule over the algebras $\bigwedge(U)$ and $\bigwedge(W)$, while the last two lines describe the operations of the elements $x \in U$ and $z \in W$.

Now let $\beta: V \times V \rightarrow K$ be a bilinear form on $V$; it determines two interior multiplications $\rfloor_{\beta}$ and $\beta L$, and a deformation $\mathrm{C} \ell(V, q ; \beta)$ of the algebra $\mathrm{C} \ell(V, q)$. This deformation is the space $\mathrm{C} \ell(V, q)$ provided with the associative multiplication $\left(b, b^{\prime}\right) \longmapsto b \star b^{\prime}$ that is characterized by (3.1), or equivalently by (3.2) (when $b$ runs through $\mathrm{C} \ell(V, q)$, and $y$ through $V$ ):

$$
\begin{array}{lll}
1 \star b=b & \text { and } & y \star b=y b+y\rfloor_{\beta} b ; \\
b \star 1=b & \text { and } & b \star y=b y+b_{\beta}\lfloor y .
\end{array}
$$

Let $q^{\prime}: V \rightarrow K$ be the quadratic form defined by $q^{\prime}(y)=q(y)+\beta(y, y)$; from $y \star y^{\prime}=y y^{\prime}+\beta\left(y, y^{\prime}\right)$, it follows that $y \star y=q^{\prime}(y)$; consequently, the identity mapping of $V$ extends to an isomorphism $\mathrm{C} \ell\left(V, q^{\prime}\right) \rightarrow \mathrm{C} \ell(V, q ; \beta)$. Because of this isomorphism, all properties of $\mathrm{C} \ell\left(V, q^{\prime}\right)$ are also valid for $\mathrm{C} \ell(V, q ; \beta)$.

Many things are invariant by deformation. The parity gradation is obviously invariant. The reversion is invariant if and only if $\beta$ is symmetric; this condition is obviously necessary since $\rho\left(y \star y^{\prime}\right)-y^{\prime} \star y=\beta\left(y, y^{\prime}\right)-\beta\left(y^{\prime}, y\right)$. The invariance of the interior multiplications is less evident; it means that

$$
\left.\left.x\rfloor_{\eta}\left(b \star b^{\prime}\right)=(x\rfloor_{\eta} b\right) \star b^{\prime}+\sigma(b) \star(x\rfloor_{\eta} b^{\prime}\right),
$$

and

$$
\left(b \star b^{\prime}\right)_{\theta}\left\lfloor z=b \star\left(b^{\prime}{ }_{\theta}\lfloor z)+\left(b_{\theta}\lfloor z) \star \sigma\left(b^{\prime}\right) .\right.\right.\right.
$$

Consequently, the deformation of $\mathrm{C} \ell(V, q ; \beta)$ by a bilinear form $\gamma: V \times V \rightarrow K$ is equal to $\mathrm{C} \ell(V, q ; \beta+\gamma)$. In particular, $\mathrm{C} \ell(V, q)$ is the deformation of $\mathrm{C} \ell(V, q ; \beta)$ by means of $-\beta$. 
If $\gamma$ is a bilinear form such that $q(y)=\gamma(y, y)$ for all $y \in V$, then $\mathrm{C} \ell(V, q ;-\gamma)$ is isomorphic to the exterior algebra $\Lambda(V)$, and it is convenient to use the symbol $\wedge$ for the multiplication in $\mathrm{C} \ell(V, q ;-\gamma)$; since $\mathrm{C} \ell(V, q)$ is the deformation of $\mathrm{C} \ell(V, q ;-\gamma)$ by means of $\gamma$, we can write:

$$
y b=y \wedge b+y\rfloor_{\gamma} b, \quad b y=b \wedge y+b_{\gamma}\llcorner y .
$$

Here is the invariance theorem for Lipschitz monoids.

Theorem 3.1 The image of $\operatorname{Lip}\left(V, q^{\prime}\right)$ by the above isomorphism $\mathrm{C} \ell\left(V, q^{\prime}\right) \rightarrow \mathrm{C} \ell(V, q ; \beta)$ is equal to $\operatorname{Lip}(V, q)$ as a subset of $\mathrm{C} \ell(V, q)$.

This theorem is the main justification of Definition 2.1. It allows to reduce the proof of many theorems (for instance Theorem 2.3) to the case $q=0$, by means of a neutral deformation $\mathrm{C} \ell(V, q ;-\gamma)$. It is also clear that, if we must find out whether a given $a \in \mathrm{C} \ell(V, q)$ is lipschitzian, we can use Theorem 2.4 to test its lipschitzian property in $\mathrm{C} \ell(V, q ;-\gamma)$; this idea leads directly to the algorithm presented in Section 4.

Theorem 3.1 is also involved in many other arguments, for instance in the proof of this other theorem (which is meaningful for any infinite field $K)$ : for each parity $p=0,1, \operatorname{Lip}_{p}(V, q)$ is an irreducible closed algebraic submanifold of dimension $1+\frac{1}{2} n(n-1)$ in $\mathrm{C} \ell_{p}(V, q)$. Moreover, for every sensible topology (the usual topology if $K$ is $\mathbb{R}$ or $\mathbb{C}$, or the Zarisky topology if $K$ is just an infinite field), $\operatorname{Lip}_{0}(V, q)$ is always the topological closure of the group $\operatorname{GLip}_{0}(V, q)$, while $\operatorname{Lip}_{1}(V, q)$ is the topological closure of $\operatorname{GLip}_{1}(V, q)$ if $q \neq 0$ (remember that $\mathrm{C} \ell_{1}(V, 0)$ contains no invertible elements).

Comments My concept of deformation stems from Chevalley (1954), who wrote the first formula (3.3), and from Bourbaki, who suggested the more general formula (3.1). The formulas (3.3) are well known when $\gamma=\mathrm{b}_{q} / 2$; but in many problems, like here, $\mathrm{b}_{q} / 2$ has very little, or even nothing, to do; after the work of the Turkish mathematician Cahit Arf (1910-1997), it is known that almost all the core of Clifford algebra theory can be covered by theorems that are valid also over a field of characteristic 2 , when $\mathrm{b}_{q} / 2$ does not exist.

My first versions of the invariance theorem are in the references (Helmstetter, 1977, 1985, 1987, 1992), where the name "Clifford monoid" is used instead of "Lipschitz monoid". But to-day, I recommend (Helmstetter, 2005) for the proofs of the theorems about Lipschitz monoids, (Helmstetter, 2011) for more information, and (Helmstetter, 2012) for a survey of the subject. A quite different version of the invariance theorem was published by Sato, Miwa and Jimbo (1978); it was preceded in 1977 by preliminary publications in Japanese.

There is a premonition of the invariance theorem in Lipschitz's work of 1880-1886; in 1880, Lipschitz used the algebra $\mathrm{C} \ell(V, q)$ of a real positive definite quadratic form $q$, without knowing that Clifford discovered it two years before him; he considered an orthogonal transformation $g$ of $(V, q)$ such that $g+\mathbf{1}$ was invertible; therefore, $g=$ $(\mathbf{1}-f)^{-1}(\mathbf{1}+f)$ for some skew symmetric operator $f$; to-day, it is convenient to use the neutral deformation $\mathrm{C} \ell\left(V, q ;-\mathrm{b}_{q} / 2\right)$, and to say that $f(z)=(u z-z u) / 2$ for some $u \in \mathrm{C} \ell^{2}\left(V, q ;-\mathrm{b}_{q} / 2\right)$; Lipschitz discovered that $g(z)=a z a^{-1}$ for some $a \in \mathrm{C} \ell_{0}(V, q)$, and explained how to derive $a$ from $u$, by means of pfaffians of skew symmetric matrices, as it is reported in the chapter 22 of (Porteous, 1995); to-day, it is easier to write $a=\exp ^{\wedge}(u)$ in $\mathrm{C} \ell\left(V, q ;-\mathrm{b}_{q} / 2\right)$.

\section{An Algorithm for Lipschitzian Elements}

An even or odd element $a$ (other than 0 ) has been given in $\mathrm{C} \ell(V, q)$ by means of its coordinates in the basis $\left(e_{J}\right)$ (with $J \in \mathcal{P}(N))$ derived from some basis $\left(e_{1}, \ldots, e_{n}\right)$ of $V$, and we must find out whether $a$ is lipschitzian.

It suffices to test the lipschitzian property of $a$ in a neutral deformation $\mathrm{C} \ell(V, q ;-\gamma)$ such that the basis $\left(e_{J}\right)$ is also the basis derived from $\left(e_{1}, \ldots, e_{n}\right)$ in the algebra $\mathrm{C} \ell(V, q ;-\gamma)$; in other words, $e_{i} \wedge e_{j}=e_{i} e_{j}$ and $\gamma\left(e_{i}, e_{j}\right)=0$ whenever $i<j$. The condition $q(y)=\gamma(y, y)$ (for all $y \in V)$ gives $\gamma\left(e_{i}, e_{i}\right)=q\left(e_{i}\right)$ and $\gamma\left(e_{i}, e_{j}\right)+\gamma\left(e_{j}, e_{i}\right)=\mathrm{b}_{q}\left(e_{i}, e_{j}\right)$ for all $i, j \in N$. Since $\gamma\left(e_{i}, e_{j}\right)=0$ if $i<j$, we must set $\gamma\left(e_{i}, e_{j}\right)=\mathrm{b}_{q}\left(e_{i}, e_{j}\right)$ if $i>j$. Now $\gamma$ is well determined; it is equal to $\mathrm{b}_{q} / 2$ if $\left(e_{1}, \ldots, e_{n}\right)$ is an orthogonal basis, but there is no pressing reason to assume that it is orthogonal. According to Theorems 3.1 and 2.4, $a$ is lipschitzian if and only if $a=d \wedge \exp ^{\wedge}(u)$ for some $u \in \mathrm{C}^{2}(V, q ;-\gamma)$, and for some element $d$ decomposable in $\mathrm{C} \ell(V, q ;-\gamma)$.

For every $r$ (an integer $\geq 0$ that has the parity of $a$ ), let $a_{\mid r}$ be the component of $a$ in $C \ell^{r}(V, q ;-\gamma)$; it is the sum of all $a_{J} e_{J}$ with $|J|=r$. If $a$ is even, and if $a_{\emptyset} \neq 0$, then $a$ is lipschitzian if and only if $a=\kappa \exp ^{\wedge}(u)$ for some $\kappa \in K$ and some $u \in \mathrm{C} \ell^{2}(V, q ;-\gamma)$; it is clear that $\kappa=a_{\emptyset}$ and $u=a_{12} / a_{\emptyset}$. With the formula (2.5) we obtain the following theorem. 
Theorem 4.1 When $a_{\emptyset} \neq 0$, then $a$ is lipschitzian if and only if

$$
a=a_{\emptyset} \exp ^{\wedge}\left(\frac{a_{\mid 2}}{a_{\emptyset}}\right) \quad \text { with } a_{\mid 2}=\sum_{|J|=2} a_{j} e_{J} .
$$

This equality in $\mathrm{C} \ell_{0}(V, q ;-\gamma)$ is equivalent to this family of equalities in $K$ :

$$
a_{\emptyset} a_{J}+\sum_{j \in J+\{i\}}(-1)^{S(\{i, j\}, J)} a_{\{i, j\}} a_{J+\{i, j\}}=0 ;
$$

there is one such equality for every $J \subset N$ of even cardinal $\geq 4$, and a particular element $i$ must be chosen in each of these subsets.

The number of equations like (4.2) is $\left(\begin{array}{c}n \\ 4\end{array}\right)+\left(\begin{array}{l}n \\ 6\end{array}\right)+\left(\begin{array}{l}n \\ 8\end{array}\right)+\cdots=2^{n-1}-1-\frac{1}{2} n(n-1)$; since it is the codimension of $\operatorname{Lip}_{0}(V, q)$ in $\mathrm{C}_{0}(V, q)$, the resulting algorithm is minimal. This algorithm requires the preliminary condition $a_{\emptyset} \neq 0$, which can be satisfied only if $a$ is even; therefore, we must propose an alternative algorithm for the other cases.

When $a \neq 0$, then $a_{F} \neq 0$ for some $F \in \mathcal{P}(N)$, and when $F \neq \emptyset$, we begin with a deformation $\mathrm{C} \ell(V, q ; \beta)$ satisfying these two conditions: firstly, the basis $\left(e_{J}\right)$ is still the basis derived from $\left(e_{1}, \ldots, e_{n}\right)$ in $\mathrm{C} \ell(V, q ; \beta)$; secondly, the basis $\left(e_{1}, \ldots, e_{n}\right)$ is orthonormal for the resulting quadratic form $q^{\prime}$ (defined by $\left.q^{\prime}(y)=q(y)+\beta(y, y)\right)$. The first condition means that $\beta\left(e_{i}, e_{j}\right)=0$ if $i<j$, so that $e_{i} \star e_{j}=e_{i} e_{j}$. Then, to make the basis $\left(e_{1}, \ldots, e_{n}\right)$ become orthonormal for $q^{\prime}$, we must set $\beta\left(e_{i}, e_{j}\right)=-\mathrm{b}_{q}\left(e_{i}, e_{j}\right)$ if $i>j$ (so that $e_{i} \star e_{j}+e_{j} \star e_{i}=0$ ), and also $\beta\left(e_{j}, e_{j}\right)=1-q\left(e_{j}\right)$ for all $j \in N$, so that $q^{\prime}\left(e_{j}\right)=1$. Now $e_{F}$ is an invertible lipschitzian element in $\mathrm{C} \ell(V, q ; \beta)$, and its inverse is $\rho_{\beta}\left(e_{F}\right)=(-1)^{S(F, F)} e_{F}$ (see (2.7)). Therefore, $a$ is lipschitzian if and only if the same is true for $b=\rho_{\beta}\left(e_{F}\right) \star a$. And since $b_{\emptyset}=a_{F} \neq 0$, we can test the lipschitzian property of $b$ with Theorem 4.1. The formula (2.6), together with $S(F, F)+S(F, J)=S(F, F+J)$, enables us to write

$$
b=(-1)^{S(F, F)} e_{F} \star a=\sum_{J}(-1)^{S(F, F+J)} a_{J} e_{F+J}=\sum_{J}(-1)^{S(F, J)} a_{F+J} e_{J} .
$$

If we apply (4.2) to $b$, and if we remember the bilinearity of $S$, we obtain the next theorem.

Theorem 4.2 When $a_{F} \neq 0$ for some $F \subset N$, then $a$ is lipschitzian if and only if the same is true for

$$
b=\sum_{J \in \mathcal{P}(N)}(-1)^{S(F, J)} a_{F+J} e_{J}
$$

and $b$ is lispchitzian if and only if

$$
a_{F} a_{F+J}+\sum_{j \in J+\{i\}}(-1)^{S(\{i, j\}, J)} a_{F+\{i, j\}} a_{F+J+\{i, j\}}=0
$$

for every subset $J$ of even cardinal $\geq 4$ (where a particular element $i$ has been freely chosen).

When $V$ has dimension 4, all Equation (4.4) lead to the Equation (0.1) mentioned in the introduction. The Equation (4.4) is still meaningful when $|J|=2$, but its meaning is trivial: $a_{F} a_{F+J}-a_{F+J} a_{F}=0$ (because $\{i, j\}=J$ ). Moreover, since $\{i\}+\{j\}$ means $\{i, j\}$ if $i \neq j$, and $\emptyset$ if $i=j$, the Equation (4.4) can also be written in this way:

$$
\sum_{j \in J}(-1)^{S(\{i\}+\{j\}, J)} a_{F+\{i\}+\{j\}} a_{F+J+\{i\}+\{j\}}=0 .
$$

When $|J|=4$, this equation does not depend on the choice of $i$ in $J$. But when $|J| \geq 6$, each choice of $i$ gives a different equation; nevertheless, if we take into account the equations corresponding to the subsets of $N$ of even cardinal between 4 and $|J|-2$, the equations associated with two choices of $i$ in $J$ are equivalent because both mean that the coordinate $b_{J}$ of $b$ agrees with the equality $b=b_{\emptyset} \wedge \exp ^{\wedge}\left(b_{\mid 2} / b_{\emptyset}\right)$.

It is clear that the Equation (4.4) or (4.5) remains the same if $F$ is replaced with $F+J$. If $i$ and $i^{\prime}$ are two elements of $J$, the Equation (4.5) also remains the same if $i$ is replaced with $i^{\prime}$, and $F$ with $F+\{i\}+\left\{i^{\prime}\right\}$.

The number of terms in (4.4) or (4.5) is $|J|$, and every term requires a multiplication of two scalars; therefore, the total number of multiplications in this algorithm is $4\left(\begin{array}{c}n \\ 4\end{array}\right)+6\left(\begin{array}{l}n \\ 6\end{array}\right)+8\left(\begin{array}{l}n \\ 8\end{array}\right)+\cdots=n\left(2^{n-2}-n+1\right)$. 


\section{Examples}

\section{First example}

Let us prove that the element $a$ in (1.1) is not lipschitzian. Since $a_{\emptyset}=0$, we must begin with the deformation $\mathrm{C} \ell(V, q ; \beta)$ that makes the basis $\left(e_{1}, \ldots, e_{n}\right)$ become orthonormal; the multiplication by $\rho_{\beta}\left(e_{1,2}\right)$ gives

$$
e_{2} \star e_{1} \star a=-1-e_{3,5}-e_{4,6}-e_{1,2,3,4}-e_{1,2,3,6}+e_{1,2,4,5}-e_{1,2,5,6}+e_{3,4,5,6} ;
$$

since the right hand member is not equal to $-\exp ^{\wedge}\left(e_{3,5}+e_{4,6}\right)$ in $\mathrm{C} \ell(V, q ;-\gamma)$, that is $-1-e_{3,5}-e_{4,6}+e_{3,4,5,6}$, we know that $a$ is not lipschitzian. We can also consider

$$
e_{4} \star e_{3} \star a=1+e_{3,5}+e_{4,6}+e_{1,2,3,4}+e_{1,2,3,6}-e_{1,2,4,5}+e_{1,2,5,6}-e_{3,4,5,6} ;
$$

again $a$ is not lipschitzian because the right hand member is not equal to $\exp ^{\wedge}\left(e_{3,5}+e_{4,6}\right)=1+e_{3,5}+e_{4,6}-e_{3,4,5,6}$.

\section{Second example}

In this example and the following ones, $V$ is a vector space of dimension 6 over $\mathbb{R}$. Let us consider this even element $a$ :

$$
\begin{aligned}
a= & 6 e_{1,2}-2 e_{1,3}+2 e_{1,5}+18 e_{2,4}+9 e_{2,6}-6 e_{3,4}-3 e_{3,6}-6 e_{4,5}+3 e_{5,6}-6 e_{1,2,3,5}-12 e_{1,2,4,6}+4 e_{1,3,4,6} \\
& +4 e_{1,4,5,6}+18 e_{2,3,4,5}-9 e_{2,3,5,6}-12 e_{1,2,3,4,5,6}
\end{aligned}
$$

Let us calculate $b=e_{2} \star e_{1} \star a$ in $\mathrm{C} \ell(V, q ; \beta)$ :

$$
\begin{aligned}
b= & 6-18 e_{1,4}-9 e_{1,6}-2 e_{2,3}+2 e_{2,5}-6 e_{3,5}-12 e_{4,6}+6 e_{1,2,3,4}+3 e_{1,2,3,6}+6 e_{1,2,4,5}-3 e_{1,2,5,6}-18 e_{1,3,4,5} \\
& +9 e_{1,3,5,6}+4 e_{2,3,4,6}+4 e_{2,4,5,6}-12 e_{3,4,5,6}
\end{aligned}
$$

let us separate the components in $\mathrm{C} \ell(V, q ;-\gamma): b=6+b_{12}+b_{\mid 4}$ since $b_{\mid 6}=0$. This $b$ is lipschitizan if and only if the equality $b=6 \exp ^{\wedge}\left(b_{12} / 6\right)$ holds in $\mathrm{C} \ell(V, q ;-\gamma)$; a straightforward calculation shows that $\frac{1}{2} b_{12} \wedge b_{12}=6 b_{14}$ and $b_{12} \wedge b_{14}=0$; it proves that $b$ is lipschitzian. Therefore, $a$ is lipschitzian too.

I emphasize the fact that this algorithm absolutely ignores $q$. Nevertheless, if we need a decomposition of $a$ into a product of vectors, we must apply a much longer and harder algorithm which cannot ignore $q$. I shall present this algorithm in a forthcoming work (entitled "Factorization of lipschitzian elements"), but here I will already show what it gives for four different choices of $q$. When $q$ coincides with $q^{\prime}$, then

$$
5 a=e_{1} e_{2}\left(e_{2}-3 e_{3}\right)\left(3 e_{1}+4 e_{4}\right)\left(e_{3}-e_{5}\right)\left(6 e_{1}-2 e_{4}-5 e_{6}\right) .
$$

When $\left(e_{1}, \ldots, e_{6}\right)$ is an orthogonal basis such that $q\left(e_{i}\right)=1$ for $i=1,2$ and $q\left(e_{i}\right)=-1$ for $i=3,4,5,6$, a similar calculation gives

$$
28 a=e_{1} e_{2}\left(e_{2}-3 e_{3}\right)\left(3 e_{1}+4 e_{4}\right)\left(3 e_{2}-5 e_{3}-4 e_{5}\right)\left(18 e_{1}+10 e_{4}-7 e_{6}\right) .
$$

When $\left(e_{1}, \ldots, e_{6}\right)$ is an orthogonal basis such that $q\left(e_{i}\right)=1$ for $i=1,2$ and $q\left(e_{i}\right)=0$ for $i=3,4,5,6$, this very degenerate $q$ leads to

$$
9 a=e_{1} e_{2}\left(e_{2}-3 e_{3}\right)\left(3 e_{1}+4 e_{4}\right)\left(3 e_{2}-10 e_{3}+e_{5}\right)\left(-6 e_{1}+10 e_{4}+9 e_{6}\right) .
$$

When $q\left(e_{i}\right)=0$ for all $i, \mathrm{~b}_{q}\left(e_{1}, e_{2}\right)=\mathrm{b}_{q}\left(e_{3}, e_{4}\right)=\mathrm{b}_{q}\left(e_{5}, e_{6}\right)=1$, and all $\mathrm{b}_{q}\left(e_{i}, e_{j}\right)$ other than these three ones vanish, then

$$
\begin{aligned}
72 a= & \left(e_{1}+e_{2}\right)\left(e_{1}+e_{2}-3 e_{3}\right)\left(2 e_{1}+3 e_{3}+4 e_{4}\right)\left(10 e_{1}-3 e_{3}+12 e_{4}\right) \\
& \left(4 e_{1}-27 e_{2}-3 e_{3}-9 e_{5}\right)\left(3 e_{2}-e_{3}+4 e_{4}+e_{5}+2 e_{6}\right) .
\end{aligned}
$$

It is much easier to find a factorization of $a$ that proves its lipschitzian property in the neutral algebra $\mathrm{C} \ell(V, 0)$; after the factorization $a_{\mid 2}=\left(2 e_{1}-6 e_{4}-3 e_{6}\right)\left(3 e_{2}-e_{3}+e_{5}\right)$, it soon appears that

$$
a=\left(2 e_{1}-6 e_{4}-3 e_{6}\right)\left(3 e_{2}-e_{3}+e_{5}\right)\left(1-e_{3,5}\right)\left(1-2 e_{4,6}\right) \text {. }
$$

\section{Third example}

$$
\begin{aligned}
a= & 2 e_{1}+e_{2}-e_{3}+2 e_{4}+e_{6}-2 e_{1,2,3}+4 e_{1,2,5}-2 e_{1,3,5}+2 e_{1,3,6}-2 e_{1,5,6}-2 e_{2,3,4}+e_{2,3,5}-4 e_{2,4,5} \\
& +e_{2,5,6}+2 e_{3,4,5}-2 e_{3,4,6}-2 e_{4,5,6}-2 e_{1,2,3,5,6}-2 e_{2,3,4,5,6}
\end{aligned}
$$


To test this odd element $a$, we consider $b=e_{1} \star a$ in $\mathrm{C} \ell(V, q ; \beta)$ :

$$
\begin{aligned}
b= & 2+e_{1,2}-e_{1,3}+2 e_{1,4}+e_{1,6}-2 e_{2,3}+4 e_{2,5}-2 e_{3,5}+2 e_{3,6}-2 e_{5,6}-2 e_{1,2,3,4}+e_{1,2,3,5}-4 e_{1,2,4,5} \\
& +e_{1,2,5,6}+2 e_{1,3,4,5}-2 e_{1,3,4,6}-2 e_{1,4,5,6}-2 e_{2,3,5,6}-2 e_{1,2,3,4,5,6} ;
\end{aligned}
$$

this $b$ (and consequently, $a$ too) is lipschitzian because $\frac{1}{2} b_{\mid 2} \wedge b_{12}=b_{\emptyset} b_{14}$ and $\frac{1}{3} b_{12} \wedge b_{14}=b_{\emptyset} b_{\mid 6}$. The equations (4.2) allow to make the verifications with a reduced number of multiplications; the 15 equations involving a subset $J$ of cardinal 4 do not depend on the choice of $i$ in $J$; but the equation with $J=N$ depends on the choice of $i$. The choice $i=4$ gives the equation

$$
b_{\emptyset} b_{N}-b_{1,4} b_{2,3,5,6}+b_{2,4} b_{1,3,5,6}-b_{3,4} b_{1,2,5,6}-b_{4,5} b_{1,2,3,6}+b_{4,6} b_{1,2,3,5}=0 ;
$$

here, this choice is clever because $b_{\{4, j\}}=0$ for $j=2,3,5,6$.

\section{Fourth example}

Here is an unusual application which shows how easily the proposed algorithm can be adapted to unexpected problems. An element $a$ of $\mathrm{C} \ell^{4}(V, 0)$ has been given, and we must find out whether $a$ is decomposable:

$$
\begin{aligned}
a= & 2 e_{1,2,3,4}+3 e_{1,2,3,6}-6 e_{1,2,4,5}-2 e_{1,2,4,6}+9 e_{1,2,5,6}-2 e_{1,3,4,5}+3 e_{1,3,5,6} \\
& -2 e_{1,4,5,6}-2 e_{2,3,4,5}+4 e_{2,3,4,6}+3 e_{2,3,5,6}-14 e_{2,4,5,6}-4 e_{3,4,5,6} .
\end{aligned}
$$

Because of Theorem 2.4, it is decomposable if and only if it is lipschitzian. Let us calculate $b=e_{4} \star e_{3} \star e_{2} \star e_{1} \star a$ :

$$
\begin{aligned}
b & =2+2 e_{1,5}-4 e_{1,6}-2 e_{2,5}+6 e_{3,5}+2 e_{3,6}+3 e_{4,6}+4 e_{1,2,5,6}-14 e_{1,3,5,6}-3 e_{1,4,5,6}+2 e_{2,3,5,6}+3 e_{2,4,5,6}-9 e_{3,4,5,6} \\
& =2+b_{12}+b_{14}
\end{aligned}
$$

since $\frac{1}{2} b_{\mid 2} \wedge b_{\mid 2}=2 b_{14}$ and $b_{\mid 2} \wedge b_{14}=0$, we conclude that $b=2 \exp ^{\wedge}\left(b_{\mid 2} / 2\right)$, that $b$ is lipschitzian, and that $a$ is decomposable in $\mathrm{C} \ell^{4}(V, 0)$.

There is an alternative algorithm, which is not much longer, and which has the advantage of giving a decomposition of $a$ when $a$ is decomposable. It uses the dual basis $\left(e_{1}^{*}, \ldots, e_{6}^{*}\right)$ of the dual space $V^{*}$, and the interior multiplication $\wedge\left(V^{*}\right) \times \mathrm{C} \ell(V, 0) \rightarrow \mathrm{C} \ell(V, 0)$. For every $\phi \in \wedge^{3}\left(V^{*}\right)$, the vector $\left.\phi\right\rfloor a$ belongs to the support of $a$ in $V$; since the coordinate $a_{1,2,3,4}$ does not vanish, it allows to find four independent vectors in the support of $a$ :

$$
\begin{aligned}
& \left.x_{1}=\left(e_{4}^{*} \wedge e_{3}^{*} \wedge e_{2}^{*}\right)\right\rfloor a=-2 e_{1}-2 e_{5}+4 e_{6}, \\
& \left.x_{2}=\left(e_{4}^{*} \wedge e_{3}^{*} \wedge e_{1}^{*}\right)\right\rfloor a=2 e_{2}-2 e_{5}, \\
& \left.x_{3}=\left(e_{4}^{*} \wedge e_{2}^{*} \wedge e_{1}^{*}\right)\right\rfloor a=-2 e_{3}-6 e_{5}-2 e_{6}, \\
& \left.x_{4}=\left(e_{3}^{*} \wedge e_{2}^{*} \wedge e_{1}^{*}\right)\right\rfloor a=2 e_{4}+3 e_{6} ;
\end{aligned}
$$

if $a$ is decomposable, $\left(x_{1}, x_{2}, x_{3}, x_{4}\right)$ is a basis of its support; consequently, $a$ is decomposable if and only if $a=\lambda x_{1} x_{2} x_{3} x_{4}$ for some scalar $\lambda$, and since $a_{1,2,3,4}=2$, it is clear that $\lambda=1 / 8$. It remains to verify that $8 a=x_{1} x_{2} x_{3} x_{4}$ in $\mathrm{C} \ell(V, 0)$.

\section{Vahlen Matrices}

Let $\left(V^{\dagger}, q^{\dagger}\right)$ be the orthogonal sum of $(V, q)$ and a plane spanned by two isotropic vectors $\varepsilon$ and $\varepsilon^{\prime}$ such that $q^{\dagger}\left(\varepsilon+\varepsilon^{\prime}\right)=1$. Thus for all $z \in V$ and all $\lambda, \mu \in K$ we can write $q^{\dagger}\left(z+\lambda \varepsilon+\mu \varepsilon^{\prime}\right)=q(z)+\lambda \mu$. The following equalities hold in $\mathrm{C} \ell\left(V^{\dagger}, q^{\dagger}\right)$ :

$$
\begin{gathered}
\varepsilon^{2}=\varepsilon^{\prime 2}=0, \quad \varepsilon \varepsilon^{\prime}+\varepsilon^{\prime} \varepsilon=1, \quad \varepsilon \varepsilon^{\prime} \varepsilon=\varepsilon, \quad \varepsilon^{\prime} \varepsilon \varepsilon^{\prime}=\varepsilon^{\prime} \\
\forall a \in \mathrm{C} \ell(V, q), \quad \varepsilon a=\sigma(a) \varepsilon, \quad \varepsilon^{\prime} a=\sigma(a) \varepsilon^{\prime} .
\end{gathered}
$$

The linear mapping $V^{\dagger} \rightarrow \operatorname{Mat}(2, \mathrm{C} \ell(V, q))$ defined by

$$
z+\lambda \varepsilon+\mu \varepsilon^{\prime} \longmapsto\left(\begin{array}{cc}
z & \lambda \\
\mu & -z
\end{array}\right)
$$


extends to an algebra isomorphism $\mathcal{M}$ from $\mathrm{C} \ell\left(V^{\dagger}, q^{\dagger}\right)$ onto $\operatorname{Mat}(2, \mathrm{C} \ell(V, q))$. It is not difficult to calculate the reciprocal isomorphism:

$$
\mathcal{M}^{-1}\left(\begin{array}{ll}
a_{1} & a_{2} \\
a_{3} & a_{4}
\end{array}\right)=\varepsilon \varepsilon^{\prime} a_{1}+a_{2} \varepsilon+\varepsilon^{\prime} a_{3}+\varepsilon^{\prime} a_{4} \varepsilon
$$

for all $a_{1}, a_{2}, a_{3}, a_{4} \in \mathrm{C} \ell(V, q)$. It follows that, for every $\alpha \in \mathrm{C} \ell\left(V^{\dagger}, q^{\dagger}\right)$,

$$
\mathcal{M}(\alpha)=\left(\begin{array}{cc}
\varepsilon \varepsilon^{\prime} \alpha \varepsilon \varepsilon^{\prime}+\varepsilon^{\prime} \sigma(\alpha) \varepsilon & \varepsilon \varepsilon^{\prime} \alpha \varepsilon^{\prime}-\varepsilon^{\prime} \sigma(\alpha) \varepsilon^{\prime} \varepsilon \\
\varepsilon \alpha \varepsilon \varepsilon^{\prime}-\varepsilon^{\prime} \varepsilon \sigma(\alpha) \varepsilon & \varepsilon \alpha \varepsilon^{\prime}+\varepsilon^{\prime} \varepsilon \sigma(\alpha) \varepsilon^{\prime} \varepsilon
\end{array}\right)
$$

The Vahlen monoid $\operatorname{Vah}(V, q)$ is the image of $\operatorname{Lip}\left(V^{\dagger}, q^{\dagger}\right)$ by this isomorphism. Many works have been devoted to the Vahlen group $\operatorname{GVah}(V, q)$ which is the image of $\operatorname{GLip}\left(V^{\dagger}, q^{\dagger}\right)$, or equivalently, the group of invertible matrices in $\operatorname{Vah}(V, q)$; and they assume $q$ to be nondegenerate. But here we are concerned neither about invertibility of matrices, nor about extra hypotheses on $q$.

If we carry the mappings $\sigma$ and $\rho \sigma$ from $\mathrm{C} \ell\left(V^{\dagger}, q^{\dagger}\right)$ into $\operatorname{Mat}(2, \mathrm{C} \ell(V, q))$ by means of the isomorphism $\mathcal{M}$, we find that

$$
\sigma\left(\begin{array}{ll}
a_{1} & a_{2} \\
a_{3} & a_{4}
\end{array}\right)=\left(\begin{array}{cc}
\sigma\left(a_{1}\right) & -\sigma\left(a_{2}\right) \\
-\sigma\left(a_{3}\right) & \sigma\left(a_{4}\right)
\end{array}\right), \quad \rho \sigma\left(\begin{array}{ll}
a_{1} & a_{2} \\
a_{3} & a_{4}
\end{array}\right)=\left(\begin{array}{cc}
\rho\left(a_{4}\right) & -\rho\left(a_{2}\right) \\
-\rho\left(a_{3}\right) & \rho\left(a_{1}\right)
\end{array}\right) .
$$

When $M$ is a Vahlen matrix, the product $M \rho \sigma(M)=\rho \sigma(M) M$ is a scalar $\operatorname{Det}(M)$ which several authors called the pseudo-determinant:

$$
\operatorname{Det}\left(\begin{array}{ll}
a_{1} & a_{2} \\
a_{3} & a_{4}
\end{array}\right)=a_{1} \rho\left(a_{4}\right)-a_{2} \rho\left(a_{3}\right)=\rho\left(a_{1}\right) a_{4}-\rho\left(a_{3}\right) a_{2} ;
$$

a Vahlen matrix $M$ is invertible if and only if $\operatorname{Det}(M) \neq 0$.

Here is the problem: and even or odd matrix is given in $\operatorname{Mat}(2, \mathrm{C} \ell(V, q))$, and we must find out whether it is a Vahlen matrix. There is an evident solution: we calculate its image in $\mathrm{C} \ell\left(V^{\dagger}, q^{\dagger}\right)$ by means of (6.1), and we apply the algorithm of Section 4 to find out whether it is lipschitzian. In $V^{\dagger}$ we can use the basis $\left(e_{1}, \ldots, e_{n}, \varepsilon, \varepsilon^{\prime}\right)$ which obliges to write the right hand member of (6.1) as $\sigma\left(a_{4}\right)+a_{2} \varepsilon+\sigma\left(a_{3}\right) \varepsilon^{\prime}+\left(a_{1}-\sigma\left(a_{4}\right)\right) \varepsilon \varepsilon^{\prime}$. This solution is correct, but here we want an algorithm that uses only the smaller algebra $\mathrm{C} \ell(V, q)$.

Some information is necessary before this algorithm is presented in Section 7. The next theorem is easy and well known.

Theorem 6.1 If M is a Vahlen matrix, we obtain another Vahlen matrix if we inflict one of these operations on it:

(1) to permute its two rows, or its two columns.

(2) to multiply the two entries of a same row, or of a same column, by the same scalar.

(3) to multiply all four entries by the same lipschitzian element, either all on the left side, or all on the right side.

Another definition is needed in the next theorems (Helmstetter, 2005).

Definition 6.2 Two lipschitzian elements $a$ and $b$ are said to be adjacent if at least one of these four conditions $(L 1),(L 2),(R 1),(R 2)$ is satisfied:

$$
\begin{aligned}
& \text { (L1) } \exists x \in V, \quad b=x a \text {; } \\
& \text { (L2) } \exists x^{\prime} \in V, \quad a=x^{\prime} b \text {; } \\
& \text { (R1) } \exists y \in V, \quad b=a y \text {; } \\
& \text { (R2) } \exists y^{\prime} \in V, \quad a=b y^{\prime} \text {. }
\end{aligned}
$$

When $a$ and $b$ are adjacent lipschitzian elements, in general all four conditions in Definition 6.2 are satisfied; but sometimes, only two of them are satisfied; the pair of satisfied conditions may be a row or a column, but never a diagonal (neither $(L 1, R 2)$ nor $(L 2, R 1)$ ). For instance, when $x$ and $y$ are isotropic vectors such that $x y \neq 0$, the adjacency between $a=y$ and $b=x y$ satisfies $(L 1, R 1)$ if $\mathrm{b}_{q}(x, y)=0$ (whence $\left.b=-y x\right)$, but it satisfies $(L 1, L 2)$ if $\mathrm{b}_{q}(x, y) \neq 0$ (because $x+y$ is invertible and $\left.b=(x+y) a\right)$.

Theorem 6.3 If $a$ is a nonzero lipschitzian element, the subset adj(a) of all lipschitzian elements adjacent to a is the image of the linear mapping $V^{*} \oplus V \rightarrow \mathrm{C} \ell(V, q)$ defined by $\left.(\ell, x) \longmapsto x a+\ell\right\rfloor a$. It is a subspace of $\mathrm{C} \ell(V, q)$ that has the same dimension as $V$.

In Theorem 6.3 we might write $a x+a\lfloor\ell$ instead of $x a+\ell\rfloor a$, because $a\lfloor\ell= \pm \ell\rfloor a$ (if $a$ is even or odd) and $x a+\sigma(a) x=x\rfloor_{q} a$ (where $\left.x\right\rfloor_{q} a$ is the interior product of $a$ by the linear form $y \longmapsto \mathrm{b}_{q}(x, y)$ ). An easy corollary of Theorem 6.3 states that adjacency is invariant by deformation. 
Corollary 6.4 If $\mathrm{C} \ell(V, q ; \beta)$ is the deformation of $\mathrm{C} \ell(V, q)$ by means of a bilinear form $\beta$, then two lipschitzian elements are adjacent in $\mathrm{C} \ell(V, q)$ if and only if they are adjacent in $\mathrm{C} \ell(V, q ; \beta)$.

All entries of a Vahlen matrix are lipschitzian; for instance, if $\alpha$ is lipschitzian and $\varepsilon \varepsilon^{\prime} \alpha \varepsilon \neq 0$, the entry $\varepsilon \varepsilon^{\prime} \alpha \varepsilon \varepsilon^{\prime}+$ $\varepsilon^{\prime} \sigma(\alpha) \varepsilon$ in (6.2) is the sum of two elements of adj $\left(\varepsilon \varepsilon^{\prime} \alpha \varepsilon\right)$, and it is lipschitzian because of Theorem 6.3. This observation can be improved.

Theorem 6.5 If the matrix $\left(a_{1}, a_{2} ; a_{3}, a_{4}\right)$ is a Vahlen matrix, then $\kappa a_{1}+v a_{4}$ and $\lambda a_{2}+\mu a_{3}$ are adjacent lipschitzian elements for all $\kappa, \lambda, \mu, v \in K$.

Theorem 6.6 If $a_{1}$ is a nonzero lipschitzian element, and if $a_{2}$ and $a_{3}$ are in $\operatorname{adj}\left(a_{1}\right)$, there are elements $a_{4} \in \mathrm{C} \ell(V, q)$ such that the matrix $\left(a_{1}, a_{2} ; a_{3}, a_{4}\right)$ is a Vahlen matrix. If $a_{4}$ is one of them, then the other ones are all $a_{4}+\theta a_{1}$ with $\theta \in K$.

The next lemma enables us to calculate $a_{4}$ when $a_{1}=1$.

Lemma 6.7 For all $x, y \in V$, the matrix $\left(\begin{array}{cc}1 & y \\ x & x y\end{array}\right)$ is a Vahlen matrix.

Indeed, the image $\alpha=x y+y \varepsilon-x \varepsilon^{\prime}+\varepsilon \varepsilon^{\prime}-x y \varepsilon \varepsilon^{\prime}$ of this matrix in $\mathrm{C} \ell\left(V^{\dagger}, q^{\dagger}\right)$ is lipschitzian; if $x$ and $y$ are colinear, this is true because $\alpha$ is in the subalgebra generated by a subspace of dimension $\leq 3$; and if they are not, the conclusion follows from the Equation (0.1) applied to the basis $\left(x, y, \varepsilon, \varepsilon^{\prime}\right)$.

\section{An Algorithm for Vahlen Matrices}

In Section 4, the algorithm for lipschitzian elements carries the problem from $\mathrm{C} \ell(V, q)$ into $\mathrm{C} \ell(V, 0)$; here, the algorithm for Vahlen matrices carries the problem from $\operatorname{Mat}(2, \mathrm{C} \ell(V, q))$ into $\operatorname{Mat}(2, \mathrm{C} \ell(V, 0))$. With every bilinear form $\beta$ on $V$, let us associate the bilinear form $\beta^{\dagger}$ on $V^{\dagger}$ defined by

$$
\beta^{\dagger}\left(x+\kappa \varepsilon+\lambda \varepsilon^{\prime}, y+\mu \varepsilon+v \varepsilon^{\prime}\right)=\beta(x, y) .
$$

The isomorphism $\mathcal{M}$ from $\mathrm{C} \ell\left(V^{\dagger}, q^{\dagger}\right)$ onto $\operatorname{Mat}(2, \mathrm{C} \ell(V, q))$ is also an isomorphism from $\mathrm{C} \ell\left(V^{\dagger}, q^{\dagger} ; \beta^{\dagger}\right)$ onto $\operatorname{Mat}(2, \mathrm{C} \ell(V, q ; \beta))$; this assertion follows from (6.1). Therefore, the Vahlen property is also invariant by deformation: an element of $\operatorname{Mat}(2, \mathrm{C} \ell(V, q))$ is a Vahlen matrix in the algebra $\operatorname{Mat}(2, \mathrm{C} \ell(V, q))$ if and only if it is a Vahlen matrix in the algebra $\operatorname{Mat}(2, \mathrm{C} \ell(V, q ; \beta))$. This property (already useful in the proof of Theorems 6.5 and 6.6) is the main piece of knowledge required by the following algorithm.

Let us consider an even matrix with entries $\left(a_{1}, a_{2} ; a_{3}, a_{4}\right)$ in $\mathrm{C} \ell(V, 0)$ such that $a_{1}$ has a nonzero scalar component. If this matrix is a Vahlen matrix, $a_{1}$ is lipschitzian and $a_{1}=\kappa \exp (u)$ for some $\kappa \in K$ and some $u \in \mathrm{C} \ell^{2}(V, 0)$. Then $a_{2}$ and $a_{3}$ are both adjacent to $a_{1}$; in the neutral algebra $C \ell(V, 0)$, these adjacencies imply equalities $a_{2}=$ $y a_{1}$ and $a_{3}=x a_{1}$ for some vectors $x$ and $y$. Because of Lemma 6.7 and Theorem 6.1 , the matrix with entries $\left(a_{1}, y a_{1} ; x a_{1}, x y a_{1}\right)$ is a Vahlen matrix; and because of Theorem 6.6, there is a scalar $\theta$ such that $a_{4}=(\theta+x y) a_{1}$. Finally, it has been proved that, for some $\kappa, \theta \in K$, for some $x, y \in V$, and for some $u \in \mathrm{C} \ell^{2}(V, 0)$,

$$
\left(\begin{array}{ll}
a_{1} & a_{2} \\
a_{3} & a_{4}
\end{array}\right)=\left(\begin{array}{cc}
\kappa \exp (u) & \kappa y \exp (u) \\
\kappa x \exp (u) & (\theta+\kappa x y) \exp (u)
\end{array}\right)
$$

The notation $a_{1 \mid k}$ means the component of $a_{1}$ in $\mathrm{C} \ell^{k}(V, 0)$; when a basis $\left(e_{1}, \ldots, e_{n}\right)$ has been chosen in $V$, then $a_{1 \mid k}$ is the sum of all $a_{1 ; J} e_{J}$ with $|J|=k$; in particular, $a_{1 \mid 0}=a_{1 ; \emptyset}$. Similar notation will be used for the other entries. The equality (7.1) allows to calculate $\kappa, \theta, x, y$ and $u$ :

$$
\kappa=a_{1 ; \emptyset}, \quad u=\frac{a_{1 \mid 2}}{a_{1 ; \emptyset}}, \quad y=\frac{a_{2 \mid 1}}{a_{1 ; \emptyset}}, \quad x=\frac{a_{3 \mid 1}}{a_{1 ; \emptyset}}, \quad \theta=a_{4 ; \emptyset} .
$$

Now the algorithm can be presented and justified. It uses the same neutral deformation $\mathrm{C} \ell(V, q ;-\gamma)$ as in Section 4 , and when it is necessary, the same auxiliary deformation $\mathrm{C} \ell(V, q ; \beta)$.

If one of the entries of the given matrix $\left(a_{1}, a_{2} ; a_{3}, a_{4}\right)$ has a nonzero scalar component, we can put this entry at the place of $a_{1}$ by means of permutations of rows or columns (see Theorem 6.1). Therefore, we can treat this case with the hypothesis $a_{1 ; \emptyset} \neq 0$. If $a_{1 ; \emptyset} \neq 0$, this matrix is a Vahlen matrix if and only if these equalities hold in the neutral deformation $\mathrm{C} \ell(V, q ;-\gamma)$ :

$$
a_{1}=a_{1 ; \emptyset} \exp ^{\wedge}\left(\frac{a_{1 \mid 2}}{a_{1 ; \emptyset}}\right), \quad a_{2}=a_{2 \mid 1} \wedge \frac{a_{1}}{a_{1 ; \emptyset}}, \quad a_{3}=a_{3 \mid 1} \wedge \frac{a_{1}}{a_{1 ; \emptyset}}, \quad a_{4}=\left(a_{1 ; \emptyset} a_{4 ; \emptyset}+a_{3 \mid 1} \wedge a_{2 \mid 1}\right) \wedge \frac{a_{1}}{\left(a_{1 ; \emptyset}\right)^{2}} .
$$


The first Equation (7.3) can be treated as it is explained in Theorem 4.1. The second and third Equations (7.3) mean that, for every even $k \geq 2$,

$$
a_{1 ; \emptyset} a_{2 \mid k+1}=a_{2 \mid 1} \wedge a_{1 \mid k}, \quad a_{1 ; \emptyset} a_{3 \mid k+1}=a_{3 \mid 1} \wedge a_{1 \mid k}
$$

For each even $k$, the Equations (7.4) give $2\left(\begin{array}{c}n \\ k+1\end{array}\right)$ equations in $K$, and each of these equations requires $k+2$ multiplications of scalars. If we take into account the second Equation (7.3), the fourth Equation (7.3) means that, for every even integer $k \geq 2$,

$$
a_{1 ; \emptyset} a_{4 \mid k}=a_{4 ; \emptyset} a_{1 \mid k}+a_{3 \mid 1} \wedge a_{2 \mid k-1} ;
$$

a similar equation $a_{1 ; \emptyset} a_{4 \mid k}=a_{4 ; \emptyset} a_{1 \mid k}+a_{3 \mid k-1} \wedge a_{2 \mid 1}$ can be deduced from the third and fourth Equations (7.3); it is equivalent to (7.5) when all Equations (7.4) are fulfilled. For every even $k$, the Equation (7.5) gives $\left(\begin{array}{l}n \\ k\end{array}\right)$ equations in $K$, and each equation needs $k+2$ multiplications in $K$. Thus the total number of equations in $K$ is

$$
\sum_{h \geq 2}\left(\begin{array}{c}
n \\
2 h
\end{array}\right)+2 \sum_{h \geq 1}\left(\begin{array}{c}
n \\
2 h+1
\end{array}\right)+\sum_{h \geq 1}\left(\begin{array}{c}
n \\
2 h
\end{array}\right)=2^{n+1}-1-\frac{1}{2}(n+2)(n+1) ;
$$

since this number is the codimension of $\operatorname{Lip}_{0}\left(V^{\dagger}, q^{\dagger}\right)$ in $\mathrm{C} \ell_{0}\left(V^{\dagger}, q^{\dagger}\right)$, this algorithm is minimal. It has the same cost as the algorithm presented in Section 4 , if we take into account that $\operatorname{dim}\left(V^{\dagger}\right)$ is $n+2$ instead of $n$; indeed, the total number of necessary multiplications of scalars is

$$
\sum_{h \geq 2} 2 h\left(\begin{array}{c}
n \\
2 h
\end{array}\right)+2 \sum_{h \geq 1}(2 h+2)\left(\begin{array}{c}
n \\
2 h+1
\end{array}\right)+\sum_{h \geq 1}(2 h+2)\left(\begin{array}{c}
n \\
2 h
\end{array}\right)=(n+2)\left(2^{n}-n-1\right) .
$$

Now we must know what can be done when the preliminary condition $a_{1 ; \emptyset} \neq 0$ is not fulfilled, or cannot be fulfilled by means of permutations of rows and columns. Since the given matrix is assumed to be $\neq 0$, we can assume that $a_{1 ; F} \neq 0$ for some subset $F \subset N$; even if $a_{1}=0$, a permutation of rows or columns can ensure this assumption. In this case, we use the deformation $\mathrm{C} \ell(V, q ; \beta)$ for which the basis $\left(e_{1}, \ldots, e_{n}\right)$ is orthonormal. We multiply all entries by $\rho_{\beta}\left(e_{F}\right)$ : we may multiply them on the let side: $b_{i}=\rho_{\beta}\left(e_{F}\right) \star a_{i}$ for $i=1,2,3,4$; but we may multiply them also on the right side: $b_{i}=a_{i} \star \rho_{\beta}\left(e_{F}\right)$ for $i=1,2,3,4$. The given matrix is a Vahlen matrix if and only if the new matrix $\left(b_{1}, b_{2} ; b_{3}, b_{4}\right)$ is a Vahlen matrix. Since $b_{1 ; \emptyset}=a_{1 ; F} \neq 0$, the new matrix can be tested by means of the previous algorithm.

\section{Examples}

\section{First example}

In all following examples, $V$ is a vector space of dimension 4 over $\mathbb{R}$. Let us begin with this odd matrix $\left(a_{1}, a_{2} ; a_{3}, a_{4}\right)$ :

$$
\left(\begin{array}{cc}
e_{2}-6 e_{3}+2 e_{1,2,3}+2 e_{2,3,4} & -4+e_{1,2}+2 e_{1,3}-8 e_{3,4}+2 e_{1,2,3,4} \\
3-e_{1,2}+6 e_{3,4}-2 e_{1,2,3,4} & e_{1}+2 e_{1,3,4}
\end{array}\right) .
$$

The permutation of the rows gives an even matrix $\left(b_{1}, b_{2} ; b_{3}, b_{4}\right)$ where $b_{1, \emptyset} \neq 0$ :

$$
\left(\begin{array}{cc}
3-e_{1,2}+6 e_{3,4}-2 e_{1,2,3,4} & e_{1}+2 e_{1,3,4} \\
e_{2}-6 e_{3}+2 e_{1,2,3}+2 e_{2,3,4} & -4+e_{1,2}+2 e_{1,3}-8 e_{3,4}+2 e_{1,2,3,4}
\end{array}\right)
$$

it is a Vahlen matrix because the Equations (7.3) are fulfilled in $\mathrm{C} \ell(V, q ;-\gamma)$ :

$$
\begin{gathered}
b_{1}=3 \exp ^{\wedge}\left(\frac{1}{3}\left(-e_{1,2}+6 e_{3,4}\right)\right), \\
3 b_{3}=\left(e_{2}-6 e_{3}\right) \wedge b_{1}, \quad 3 b_{2}=e_{1} \wedge b_{1}, \\
9 b_{4}=\left(-12+\left(e_{2}-6 e_{3}\right) \wedge e_{1}\right) \wedge b_{1} .
\end{gathered}
$$

Alternatively, we can also use $\mathrm{C} \ell(V, q ; \beta)$ and test the matrix

$$
\left(\begin{array}{cc}
1-2 e_{1,3}-6 e_{2,3}+2 e_{3,4} & -e_{1}-4 e_{2}-2 e_{1,2,3}-2 e_{1,3,4}-8 e_{2,3,4} \\
e_{1}+3 e_{2}+2 e_{1,3,4}+6 e_{2,3,4} & -e_{1,2}-2 e_{1,2,3,4}
\end{array}\right)
$$


with entries $b_{i}^{\prime}=e_{2} \star a_{i}$ (for $i=1,2,3,4$ ); it is a Vahlen matrix because

$$
\begin{gathered}
b_{1}^{\prime}=\exp ^{\wedge}\left(-2 e_{1,3}-6 e_{2,3}+2 e_{3,4}\right), \\
b_{3}^{\prime}=\left(e_{1}+3 e_{2}\right) \wedge b_{1}^{\prime}, \quad b_{2}^{\prime}=\left(-e_{1}-4 e_{2}\right) \wedge b_{1}^{\prime}, \\
b_{4}^{\prime}=\left(e_{1}+3 e_{2}\right) \wedge\left(-e_{1}-4 e_{2}\right) \wedge b_{1}^{\prime} .
\end{gathered}
$$

\section{Second example}

$$
\left(\begin{array}{cr}
-e_{1,2}-3 e_{1,3}+e_{1,4}+7 e_{2,3} & 2 e_{1}-4 e_{2}+2 e_{3}+10 e_{1,2,3} \\
-2 e_{2,4}+e_{3,4}+9 e_{1,2,3,4} & +3 e_{1,2,4}+e_{1,3,4}-5 e_{2,3,4} \\
e_{1}-2 e_{2}+e_{3}+10 e_{1,2,3} & -3 e_{1,2}+e_{1,3}+e_{2,3}-3_{1,2,3,4} \\
+e_{1,3,4}-2 e_{2,3,4} &
\end{array}\right)
$$

Let us permute the rows, and multiply all entries by $e_{1}$ on the left side in the auxiliary algebra $\mathrm{C} \ell(V, q ; \beta)$ :

$$
\left(\begin{array}{cc}
1-2 e_{1,2}+e_{1,3}+10 e_{2,3} & -3 e_{2}+e_{3}+e_{1,2,3}-3 e_{2,3,4} \\
+e_{3,4}-2_{1,2,3,4} & \\
-e_{2}-3 e_{3}+e_{4}+7 e_{1,2,3} & 2-4 e_{1,2}+2_{1,3}+10 e_{2,3} \\
-2 e_{1,2,4}+e_{1,3,4}+9_{2,3,4} & +3 e_{2,4}+e_{3,4}-5 e_{1,2,3,4}
\end{array}\right)
$$

Let $\left(b_{1}, b_{2} ; b_{3}, b_{4}\right)$ be this matrix. It is a Vahlen matrix because the equalities (7.3) prove to be true in $\mathrm{C} \ell(V, q ;-\gamma)$ :

$$
\begin{gathered}
b_{1}=\exp ^{\wedge}\left(-2 e_{1,2}+e_{1,3}+10 e_{2,3}+e_{3,4}\right), \\
b_{3}=\left(-e_{2}-3 e_{3}+e_{4}\right) \wedge b_{1}, \quad b_{2}=\left(-3 e_{2}+e_{3}\right) \wedge b_{1}, \\
b_{4}=\left(2+\left(-e_{2}-3 e_{3}+e_{4}\right) \wedge\left(-3 e_{2}+e_{3}\right)\right) \wedge b_{1} .
\end{gathered}
$$

In general, the Equations (4.2), (7.4) and (7.5) allow to verify the Equations (7.3) with a reduced number of multiplications; here there are two Equations (7.5):

$$
b_{1 ; \emptyset} b_{4 \mid 2}=b_{4 ; \emptyset} b_{1 \mid 2}+b_{3 \mid 1} \wedge b_{2 \mid 1} \quad \text { and } \quad b_{1 ; \emptyset} b_{4 \mid 4}=b_{4 ; \emptyset} b_{1 \mid 4}+b_{3 \mid 1} \wedge b_{2 \mid 3}
$$

Third example

$$
\left(\begin{array}{cr}
1+e_{1,2}-e_{1,3}+e_{1,4}-e_{2,3} & -2 e_{2}+2 e_{3}+2 e_{1,2,4}-2 e_{1,3,4} \\
+e_{2,4}-e_{3,4}-e_{1,2,3,4} & \\
e_{3}-e_{4}+e_{1,2,3}-e_{1,2,4} & 1+e_{1,2}-e_{1,3}+e_{1,4}+e_{2,3} \\
-e_{2,4}+e_{3,4}+e_{1,2,3,4}
\end{array}\right)
$$

This matrix $\left(a_{1}, a_{2} ; a_{3}, a_{4}\right)$ is a Vahlen matrix because

$$
\begin{gathered}
a_{1}=\exp ^{\wedge}\left(e_{1,2}-e_{1,3}+e_{1,4}-e_{2,3}+e_{2,4}-e_{3,4}\right), \\
a_{3}=\left(e_{3}-e_{4}\right) \wedge a_{1}, \quad a_{2}=\left(-2 e_{2}+2 e_{3}\right) \wedge a_{1}, \\
a_{4}=\left(1+\left(e_{3}-e_{4}\right) \wedge\left(-2 e_{2}+2 e_{3}\right)\right) \wedge a_{1} .
\end{gathered}
$$

I emphasize the fact that this algorithm completely ignores the initial quadratic form $q$. In this last example, if $q$ is the quadratic form for which $\left(e_{1}, e_{2}, e_{3}, e_{4}\right)$ is an orthogonal basis such that $q\left(e_{1}\right)=q\left(e_{2}\right)=q\left(e_{3}\right)=-1$ and $q\left(e_{4}\right)=1$, then none of the four entries of the given matrix is invertible; nevertheless, this matrix is invertible because its pseudo-determinant (defined in (6.3)) is 8 . For this algorithm, the absence of an invertible entry raises no difficulty.

\section{References}

Bourbaki, N. Algèbre, chapitre IX. Paris, Hermann.

Chevalley, C. (1954). The algebraic theory of spinors. New York: Columbia Univ. Press. Reprinted in C. Chevalley (1997), Collected works, vol.2, Springer.

Helmstetter, J. (1977). Groupe de Clifford pour des formes quadratiques dégénérées. C. R. Acad. Sc. Paris A, 285, 175-177. 
Helmstetter, J. (1985). Algèbres de Clifford et algèbres de Weyl. Cahiers Math. Montpellier 34, Université de Montpellier.

Helmstetter, J. (1987). Monoïdes de Clifford et déformations d'algèbres de Clifford. J. of Algebra, 111, 14-48. http://dx.doi.org/10.1016/0021-8693(87)90241-9

Helmstetter, J. (1992). Clifford groups for arbitrary quadratic forms. In A. Micali, R. Boudet, \& J. Helmstetter (Eds.), Clifford Algebras and their Applications in Mathematical Physics, Fundamental Theories of Physics, 47, 33-38. Dordrecht, Kluwer Ac. Publishers. http://dx.doi.org/10.1007/978-94-015-8090-8_4

Helmstetter, J. (2005). Lipschitz monoids and Vahlen matrices. Adv. Applied Clifford Algebras, 15, 83-122. http://dx.doi.org/10.1007/s00006-005-0006-z

Helmstetter, J. (2011). Lipschitzian subspaces in Clifford algebras. J. of Algebra, 328, 461-483. http://dx.doi.org/10.1016/j.algebra.2010.09.29

Helmstetter, J. (2012). A survey of Lipschitz monoids. Adv. Applied Clifford Algebras, 22(3), 665-688. http://dx.doi.org/10.1007/s00006-012-0354-4

Helmstetter, J., \& Micali, A. (2008). Quadratic mappings and Clifford algebras. Birkhäuser.

Porteous, I. (1995). Clifford algebras and the classical groups. Cambridge Univ. Press. http://dx.doi.org/10.1017/CBO9780511470912

Sato, M., Miwa, T., \& Jimbo, M. (1978). Holonomic quantum fields I. Publ. R. I. M. S., Kyoto Univ., 14, 223-267. http://dx.doi.org/10.2977/prims/1195189284

\section{Copyrights}

Copyright for this article is retained by the author(s), with first publication rights granted to the journal.

This is an open-access article distributed under the terms and conditions of the Creative Commons Attribution license (http://creativecommons.org/licenses/by/3.0/). 